\title{
A NOTE ON STABLE ITERATED FUNCTION SYSTEMS
}

\author{
ROY A. MIMNA AND THOMAS SMOTZER \\ Received 30 November 2005; Revised 20 April 2006; Accepted 4 May 2006
}

The properties of continuous stable iterated function systems are investigated, and equicontinuity is studied in relation to stable iterated function systems.

Copyright (c) 2006 Hindawi Publishing Corporation. All rights reserved.

\section{Introduction and preliminaries}

Let $X$ denote a compact metric space with metric $d$, and let $f: X \rightarrow X$ denote a continuous self-map on $X$. For any subset $E$ of $X$, we let $C l(E)$ denote the closure of $E$. Following $[1]$, we denote by $\{\mathfrak{I}, X\}$ an iterated function system, or IFS, on $X$. That is, $\mathfrak{I}$ is a finite family $\left\{f_{1}, \ldots, f_{m}\right\}$ of continuous self-maps on $X$. In this paper we do not consider the case in which $\mathfrak{I}$ is an infinite family. Let $\mathfrak{J}^{\infty}$ denote the family of infinite sequences of compositions of functions in $\mathfrak{J}$. That is, a given sequence $F$ in $\mathfrak{J}^{\infty}$ is composed of arbitrary compositions of elements of $\mathfrak{I}$, so that for each positive integer $n, F_{n}=f_{1} \circ f_{2} \circ \cdots \circ f_{n}$, where each $f_{i}, 1 \leq i \leq n$, is an element of $\{\mathfrak{I}, X\}$.

An IFS $\{\mathfrak{I}, X\}$ is hyperbolic if there is a real number $\lambda$, where $0<\lambda<1$, such that $d(f(x), f(y)) \leq \lambda * d(x, y)$ for all points $x$ and $y$ in $X$ and for all $f \in \mathfrak{J}$. An IFS $\{\mathfrak{J}, X\}$ is stable if for every infinite sequence $F, \operatorname{diam}\left(F_{n}(X)\right) \rightarrow 0$ as $n \rightarrow \infty$. Equivalently, $\{\mathfrak{I}, X\}$ is stable if $\left\{F_{n}(X)\right\}_{n \geq 1}$ has a one-point set as its limit. If $\{\mathfrak{I}, X\}$ consists of a single function $f$, then $\{\mathfrak{I}, X\}$ is stable if $\operatorname{diam}\left(f^{k}(X)\right) \rightarrow 0$ as $k \rightarrow \infty$, where $f^{k}$ is the $k$-fold composition of $f$. By the definition of stability, each sequence $F^{\lambda}$ in $\mathfrak{J}^{\infty}$, where $\lambda \in \Lambda$ for some indexing set $\Lambda$, converges to a unique point $x_{\lambda}$. Let $\mathbf{F}$ denote the sequence of nested decreasing compact sets formed by the family of sequences $\left\{F_{n}^{\lambda}(X)\right\}_{\lambda \in \Lambda}$. For example, in the wellknown IFS $\left\{f_{1}, f_{2},[0,1]\right\}$, where $f_{1}(x)=(1 / 3) x$ and $f_{2}(x)=(1 / 3) x+2 / 3, \mathbf{F}$ is the nested sequence of closed subintervals of $[0,1]$ converging to the Cantor ternary set.

An IFS $\{\mathfrak{I}, X\}$ is conjugate to an IFS $\left\{\mathfrak{I}^{\prime}, X^{\prime}\right\}$ if there exists a homeomorphism $h: X \rightarrow$ $X^{\prime}$ such that $\mathfrak{I}^{\prime}=h \circ \mathfrak{J} \circ h^{-1}=\left\{h \circ f \circ h^{-1}: f \in \mathfrak{J}\right\}$. The conjugacy then assures that the qualitative dynamic behavior of the systems $\mathfrak{I}$ and $\mathfrak{J}^{\prime}$ are largely the same. Clearly, stable systems exist which are not themselves hyperbolic, but which are conjugate to hyperbolic systems. In [1] it is shown that functions which are either continuous and monotone or 
belong to the Lip $[0,1]$ class are conjugate to hyperbolic functions. Moreover, there exists a continuous stable IFS which is not conjugate to any hyperbolic IFS (see [1]). Although noncontinuous stable IFSs exist, we confine our investigation to the case where all $f_{i}$ in $\{\mathfrak{I}, X\}$ are continuous. We refer to such systems as "continuous stable IFSs." We consider some of the properties of continuous stable systems, including systems which are neither hyperbolic nor conjugate to any hyperbolic system. As observed in [1], continuous stable IFSs which are not hyperbolic and are not conjugate to any hyperbolic system are residual in the set of all stable IFSs.

\section{Properties of continuous stable iterated function systems}

We now consider an IFS $\{\mathfrak{I}, X\}$ which is made up of a finite number of self-maps, each of which, taken individually, is stable. It turns out that such an iterated function system is not necessarily a stable IFS.

Proposition 2.1. An iterated function system $\{\mathfrak{J}, X\}$, where $\mathfrak{I}$ is a finite family $\left\{f_{1}, \ldots, f_{m}\right\}$ of self-maps, each of which, taken individually, is stable, need not be a stable IFS.

Proof. Let

$$
f(x)= \begin{cases}\frac{1}{8} & \text { if } 0 \leq x \leq \frac{1}{2} \\ 2 x-\frac{7}{8} & \text { if } \frac{1}{2} \leq x \leq \frac{3}{4} \\ \frac{5}{8} & \text { if } \frac{3}{4} \leq x \leq 1\end{cases}
$$

and let

$$
g(x)= \begin{cases}\frac{5}{8} & \text { if } 0 \leq x \leq \frac{1}{8}, \\ -\left(\frac{1}{2}\right) x+\frac{11}{16} & \text { if } \frac{1}{8} \leq x \leq \frac{5}{8} \\ \frac{3}{8} & \text { if } \frac{5}{8} \leq x \leq 1 .\end{cases}
$$

Then

$$
g[f(x)]= \begin{cases}\frac{5}{8} & \text { if } 0 \leq x \leq \frac{1}{2} \\ \frac{9}{8}-x & \text { if } \frac{1}{2} \leq x \leq \frac{3}{4} \\ \frac{3}{8} & \text { if } \frac{3}{4} \leq x \leq 1\end{cases}
$$

Taken as single functions, both $f$ and $g$ are stable. However, when we take the composition $g \circ f \circ g \circ f$, we obtain the following function which cannot have a unique fixed 
point under further iteration:

$$
g \circ f \circ g \circ f(x)= \begin{cases}\frac{1}{2} & \text { if } 0 \leq x \leq \frac{1}{2}, \\ x & \text { if } \frac{1}{2} \leq x \leq \frac{5}{8} \\ \frac{5}{8} & \text { if } \frac{5}{8} \leq x \leq 1 .\end{cases}
$$

The conclusion now follows.

Recall that for any compact metric space $X$ with metric $d$, we may define the Hausdorff metric $h$ on the space $\hat{H}(X)$ of closed subsets of $X$ so that for any "points" $C$ and $E$ in the space $\hat{H}(X), h(C, E)=\min \left\{\delta>0: C \subset B_{\delta}(E)\right.$ and $\left.E \subset B_{\delta}(C)\right\}$, where $B_{\delta}(C)$ and $B_{\delta}(E)$ are open $\delta$-balls about $C$ and $E$, respectively. Equivalently, $h(C, E)=\max [\max \{d(x, E)$ : $x \in C\} ; \max \{d(C, x): x \in E\}]$. It is well known that if $(X, d)$ is compact, then $(\hat{H}(X), h)$ is also compact. Let $\{\mathfrak{J}, X\}=\left\{f_{1}, \ldots, f_{m}, X\right\}$ be an IFS and define the function $F: \hat{H}(X) \rightarrow$ $\hat{H}(X)$, so that $F(C)=\bigcup_{i=1}^{m} f_{i}(C)$ for every $C \in \hat{H}(X)$. Then consider the sequence of iterates $F^{k}(\hat{H}(X))$ in the metric space $(\hat{H}(X), h)$, so that $F^{k}(\hat{H}(X))=F\left(F^{k-1}(\hat{H}(X))\right)$ for $k=1,2, \ldots$ If the IFS $\{F, \hat{H}(X)\}$ is hyperbolic, it is well known that there exists a unique nonempty compact invariant set $A$, where $A=F(A)=\bigcup_{i=1}^{m} f_{i}(A)$, so that $\left\{F^{k}(X)\right\}_{k=1}^{\infty}$ converges to $A$ in the Hausdorff metric. This result has also been proved for systems conjugate to hyperbolic systems (see [3, page 114]). In Theorem 2.3 below we prove a similar result for iterated function systems which are continuous and stable. First, we prove a result on the continuity of the function $F: \hat{H}(X) \rightarrow \hat{H}(X)$.

Proposition 2.2. Let $\{\mathfrak{I}, X\}=\left\{f_{1}, \ldots, f_{m}, X\right\}$ be a continuous stable IFS on a compact metric space $(X, d)$. Let $(\hat{H}(X), h)$ denote the space of all closed subsets of $X$ with the Hausdorff metric $h$. Then the function $F: \hat{H}(X) \rightarrow \hat{H}(X)$, where $F$ is defined so that $F(C)=\bigcup_{i=1}^{m} f_{i}(C)$ for all $C$ in $\hat{H}(X)$, is continuous in the Hausdorff metric.

Proof. Let $\epsilon>0$ be given. Let $A \in \hat{H}(X)$ be any point in the domain of $F$. Let $B(F(A), \epsilon)$ be an $\epsilon$-ball about $F(A)$ in the Hausdorff metric. We show that there exists $\delta>0$ such that $F(B(A), \delta) \subset B(F(A), \epsilon)$. Observe that since each $f_{i}$ is uniformly continuous on the compact space $X$, there exists $\delta_{i}>0$ for each $f_{i}$, so that $d\left(x_{1}, x_{2}\right)<\delta_{i}$ implies that $d\left(f_{i}\left(x_{1}\right)\right.$, $\left.f_{i}\left(x_{2}\right)\right)<\epsilon$, for all $x_{1}, x_{2} \in X$. Let $\delta=\min _{1 \leq i \leq m}\left\{\delta_{i}\right\}$. To show that $F(B(A), \delta) \subset B(F(A), \epsilon)$, let $C$ be any point in $B(A, \delta)$. If $A \cap C$ is not empty in the topology of the underlying metric $d$, then all points in $(C \cap A)$ are mapped into $B(F(A), \epsilon)$. Now consider all points in $C$ in the metric $d$ which are not in $A$. Every such point is $\delta$-close to some point in $A$ in the $d$-metric, because $h(A, C)=\min \left\{\delta>0: C \subset B_{\delta}(A) ; A \subset B_{\delta}(C)\right\}$. By the uniform continuity of the $f_{i}$, it follows that every point in $F(C)$ is $\epsilon$-close to some point in $F(A)$. By a similar argument, every point in $F(A)$ is $\epsilon$-close to some point in $F(C)$. Then by the definition of the Hausdorff metric, $C \in B(F(A), \epsilon)$. Hence, $F(B(A), \delta) \subset B(F(A), \epsilon)$, and $F$ is continuous on $(\hat{H}(X), h)$.

Theorem 2.3. Let $\{\mathfrak{I}, X\}=\left\{f_{1}, \ldots, f_{m}, X\right\}$ be a continuous stable IFS on a compact metric space $(X, d)$. Let $(\hat{H}(X), h)$ denote the space of all closed subsets of $X$ with the Hausdorff 
metric $h$. Then the sequence $\left\{F^{n}(\hat{H}(X))\right\}_{n=1}^{\infty}$ converges to a point $A$ in $(\hat{H}(X), h)$. That is, the iterated function system $\{F, \hat{H}(X)\}$ is (continuous and) stable.

Proof. First, observe that the iterates $\left\{F^{n}(\hat{H}(X))\right\}_{n=1}^{\infty}$ are nested. To show $\left\{F^{n}(\hat{H}(X))\right\}_{n=1}^{\infty}$ converges in the Hausdorff metric to a point $A \in \hat{H}(X)$, we show that $\bigcap_{n=1}^{\infty} F^{n}(\hat{H}(X))=$ $A$. Since $\hat{H}(X)$ is compact and $F$ is continuous, then $F^{n}(\hat{H}(X))=A_{n}$ is compact for every $n$. For each $n, n=1,2, \ldots$, let $a_{n} \in A_{n}$ be a point in the underlying metric space $(X, d)$. Then the sequence $\left\{a_{n}\right\}_{n=1}^{\infty}$ is contained in the set $A_{1}$. Since $A_{1}$ is compact, there is a convergent subsequence $\left\{a_{n_{k}}\right\}_{k=1}^{\infty}$ with limit $a$ in $A_{1}$. But that subsequence, except for possibly its first element, is also contained in $A_{2}$, and since $A_{2}$ is compact, the limit must be contained in $A_{2}$. By induction, the limit is contained in every $A_{n}$. Hence, the limit $a$ is contained in the intersection $A=\bigcap_{n=1}^{\infty} F^{n}(\hat{H}(X))$, and $A$ is not empty. Observe that $A$ is compact and thus is a point in $\hat{H}(X)$. The above argument also shows that $\left\{F^{n}(\hat{H}(X))\right\}_{n=1}^{\infty}$ converges to $A$ in the Hausdorff metric.

We say that the map $f: X \rightarrow X$ has equicontinuous iterates if for any $x \in X$ and for any $\epsilon>0$ there exists a $\delta>0$ such that $d\left(f^{n}(x), f^{n}(y)\right)<\epsilon$ whenever $d(x, y)<\delta$ for all $y \in X$ and for all positive integers $n$. Since $X$ is compact, every transformation on $X$ with equicontinuous iterates has equiuniformly continuous iterates. That is, for any $\epsilon>0$, there exists $\delta>0$ such that $d(x, y)<\delta$ implies that $d\left(f^{n}(x), f^{n}(y)\right)<\epsilon$ for all positive integers $n$ and for all $x$ and $y$ in $X$. Clearly, all hyperbolic maps of the form $f: X \rightarrow X$ have equiuniformly continuous iterates. Also, observe that since a stable system $\{f, X\}$ converges to a one-point set, we may think of this convergence as convergence of the functions $f_{1}, f_{2}, \ldots, f_{k}, \ldots$, to a constant function $f$. We let $S(X, X)$ denote the set of all continuous stable self-maps with the supremum metric $\rho$; that is, each element in $S(X, X)$ is a self-map of the form $f: X \rightarrow X$, and for any two distinct elements $f$ and $g$ in $S(X, X)$, $\rho(f, g)=\sup _{x \in X}\{d(f(x), g(x)): x \in X\}$. We now show that a necessary condition for a map $f$ to be continuous and stable is that $f$ has equiuniformly continuous iterates.

Proposition 2.4. Let $f$ be an element of $S(X, X)$. Then the iterates of $f$ are equiuniformly continuous.

Proof. Fix $\epsilon>0$. Let $\{f, X\}$ be a continuous stable IFS comprised of a single function. Since $\left\{f^{n}\right\}_{n=1}^{\infty}$ converges to a constant function $g=z$, for some $z \in X$, then there is a positive integer $N$ such that $d\left(f^{n}(x), z\right)<\epsilon / 2$ and $d\left(f^{n}(y), z\right)<\epsilon / 2$, and hence $d\left(f^{n}(x)\right.$, $\left.f^{n}(y)\right)<\epsilon$, for all $n \geq N$ and for all $x, y$ in $X$. Since $f^{n}$ is uniformly continuous for every $n$, then there exists $\delta_{n}>0$ such that $d(x, y)<\delta_{n}$ implies that $d\left(f^{n}(x), f^{n}(y)\right)<\epsilon$ for each $n$, $1 \leq n<N$. Then for $\delta=\min \left\{\delta_{n}: 1 \leq n<N\right\}, d(x, y)<\delta$ implies that $d\left(f^{n}(x), f^{n}(y)\right)<\epsilon$ for all $n, 1 \leq n<N$, and for all $x, y$ in $X$. Hence, $f$ has equiuniformly continuous iterates.

The reader will easily find examples to show that the converse of Proposition 2.4 is not true.

Corollary 2.5. Each sequence $\left\{F_{n}(X)\right\}_{n=1}^{\infty}$ in a continuous stable iterated function system $\{\mathfrak{J}, X\}$ is made up of functions, which, taken together as a single sequence, make up a set of equiuniformly continuous functions. 
Corollary 2.6. Let $f \in S(X, X)$. Then $f$ is not surjective.

Proof. Since $f \in S(X, X)$, then by Proposition 2.4, $f$ has equiuniformly continuous iterates. By [2, Corollary 8], any surjective self-map $f$ on a compact metric space whose iterates are equicontinuous is a homeomorphism. If $f$ is a homeomorphism, then so are all of the iterates of $f$. But it then follows that the iterates of $f(X)$ do not converge to a single point, a contradiction.

Remark 2.7. The proof of Proposition 2.4 shows that the sequence $\left\{f^{n}\right\}_{n=1}^{\infty}$ converges uniformly to the constant map $g$. That is, for any $\epsilon>0$, there exists a positive integer $N$, so that for all $n \geq N$, and for all $x \in X, \rho\left(f^{n}, g\right)<\epsilon$.

Proposition 2.8. Let $\{\mathfrak{J}, X\}=\left\{f_{1}, \ldots, f_{m}, X\right\}$ be a continuous stable IFS on a compact metric space $(X, d)$. Let $(\hat{H}(X), h)$ denote the space of all closed subsets of $X$ with the Hausdorff metric $h$. Then the function $F$ on $\hat{H}(X)$, as defined in Theorem 2.3 above, has equiuniformly continuous iterates.

Proof. By Theorem 2.3 above, the system $\{F, \hat{H}(X)\}$ is stable. Now by mimicking the proof of Proposition 2.4 above, the result follows.

\section{References}

[1] A. Ambroladze, K. Markström, and H. Wallin, Stability versus hyperbolicity in dynamical and iterated function systems, Real Analysis Exchange 25 (1999/2000), no. 1, 449-461.

[2] A. M. Bruckner and T. Hu, Equicontinuity of iterates of an interval map, Tamkang Journal of Mathematics 21 (1990), no. 3, 287-294.

[3] P. F. Duvall Jr., J W. Emert, and L. S. Husch, Iterated function systems, compact semigroups, and topological contractions, Continuum Theory and Dynamical Systems, Lecture Notes in Pure and Applied Mathematics, vol. 149, Dekker, New York, 1993, pp. 113-155.

Roy A. Mimna: Department of Mathematics, Youngstown State University, Youngstown, OH 44555, USA

E-mail address: ramimna@ysu.edu

Thomas Smotzer: Department of Mathematics, Youngstown State University, Youngstown, $\mathrm{OH} 44555$, USA

E-mail address: tsmotzer@math.ysu.edu 


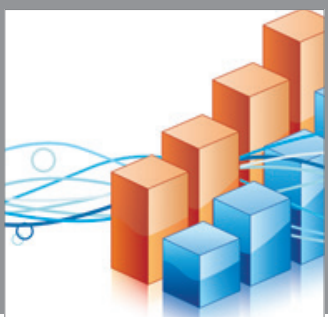

Advances in

Operations Research

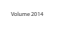

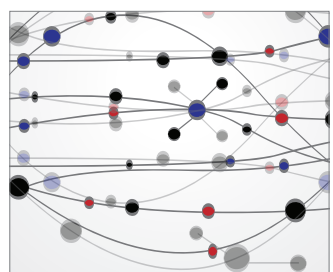

\section{The Scientific} World Journal
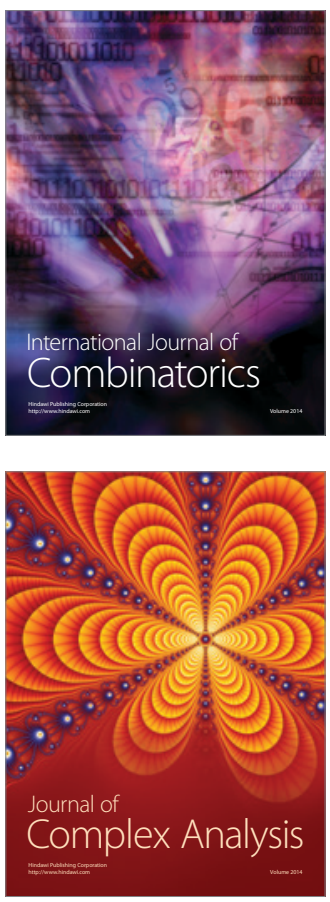

International Journal of

Mathematics and

Mathematical

Sciences
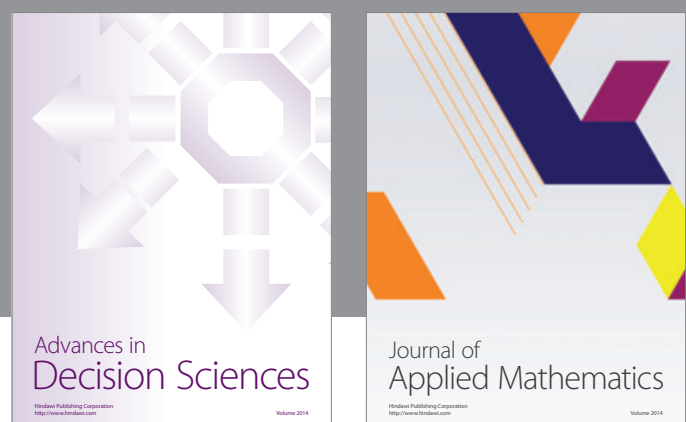

Journal of

Applied Mathematics
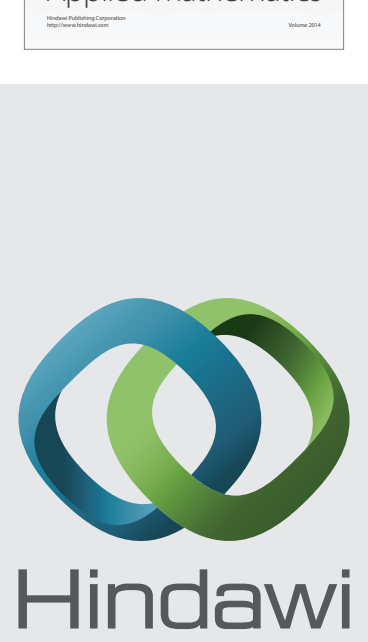

Submit your manuscripts at http://www.hindawi.com
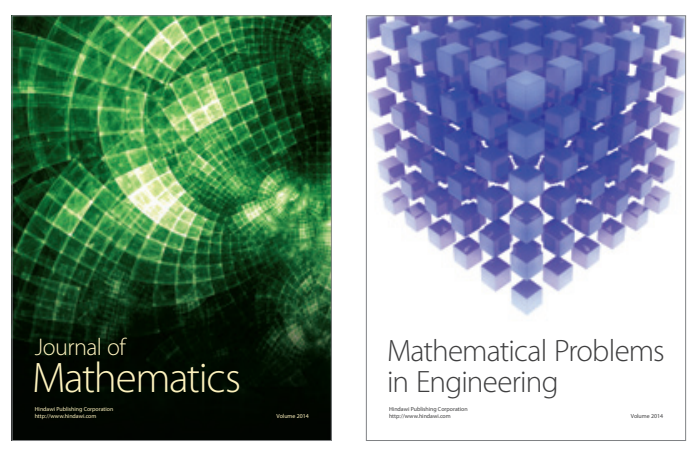

Mathematical Problems in Engineering
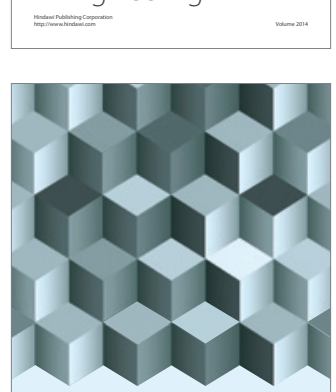

Journal of

Function Spaces
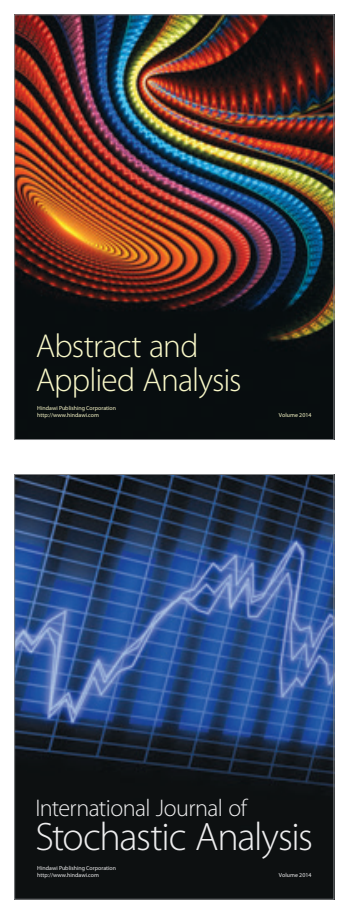

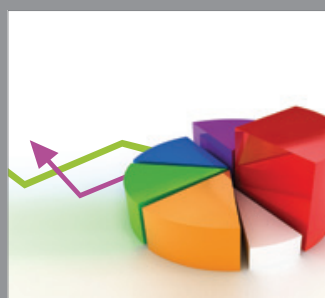

ournal of

Probability and Statistics

Promensencen
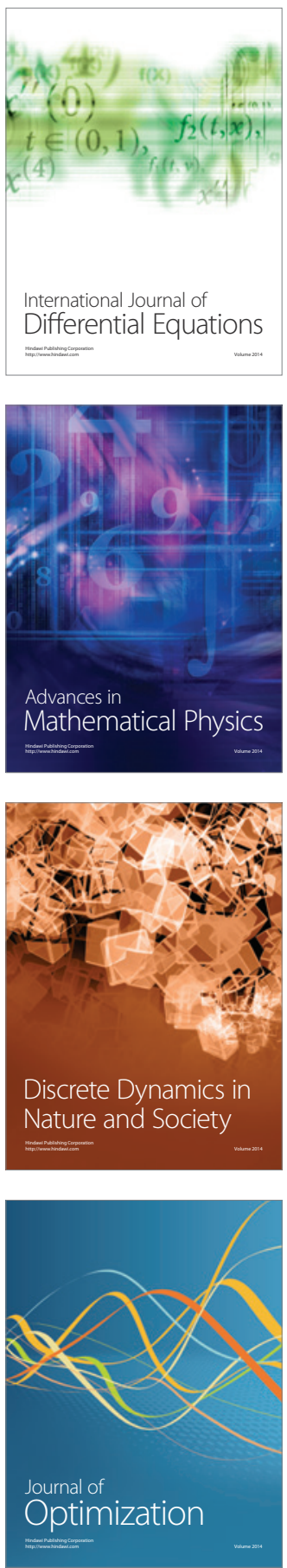